\title{
Joint Transmit and Receive Antenna Selection in MIMO Systems Based on Swarm Intelligence Algorithm
}

\section{Zhang Yiwen}

Jimei University

\section{Su Sunqing}

Jimei University

Liao Wenliang

Jimei University

Lei Guowei ( $\nabla$ gwlei@163.com )

Jimei University https://orcid.org/0000-0001-8902-1091

\section{Yang Guangsong}

Jimei University

\section{Research Article}

Keywords: JTRAS, fractional coding, cat swarm optimization, genetic algorithm, particle swarm optimization

Posted Date: June 29th, 2021

DOI: https://doi.org/10.21203/rs.3.rs-553146/v1

License: (c) (1) This work is licensed under a Creative Commons Attribution 4.0 International License. Read Full License 


\title{
Joint transmit and receive antenna selection in MIMO systems based on swarm intelligence algorithm
}

\author{
Zhang Yiwen ${ }^{1,2}$, Su Sunqing ${ }^{1}$, Liao Wenliang ${ }^{1}$, Lei Guowei ${ }^{1 *}$, Yang Guangsong ${ }^{3}$ \\ ${ }^{1}$ School of Science, Jimei university, Xiamen 361021, P.R. China \\ ${ }^{2}$ Department of Electronic and Electrical Engineering, the university of Sheffield, United Kingdom \\ ${ }^{3}$ School of Information Engineering, Jimei university, Xiamen 361021, P.R. China \\ *corresponding author: gwlei@163.com
}

\begin{abstract}
In multiple-input-multiple-output (MIMO) systems, the selection of receive and transmit antennas is not just effective in increasing system capacity, but also in reducing RF link costs and system complexity. The exhaustive algorithm, i.e. the joint transmit and receive antenna selection (JTRAS) with the best accuracy, can search all the subsets of both transmit and receive antennas in order to find the optimal solution. However, with the increase of the number of antennas, the computational complexity is too large and its applicability is limited. In this paper, the antennas are coded by fractional coding with the maximization of channel capacity as the basic criterion, and three intelligent algorithms, namely genetic algorithm, cat swarm algorithm and particle swarm algorithm, are applied for antenna selection. The simulation results demonstrate that all three algorithms can efficiently accomplish the antenna selection. In the end, we compare them in terms of speed, accuracy and complexity of the search in MIMO systems.
\end{abstract}

Keywords: JTRAS, fractional coding, cat swarm optimization, genetic algorithm, particle swarm optimization

\section{Introduction}

MIMO (Multiple-Input-Multiple-Output) systems can exponentially enhance the capacity of communication systems ${ }^{[1-2]}$ and the linkage of wireless transmission. Moreover, it can greatly improve system performance without the sacrifice of bandwidth compared to single-input and single-output systems ${ }^{[3-5]}$. And multiple antennas, space-time coding and other techniques can significantly improve spectrum utilization, which is a major breakthrough in smart antenna technology in mobile communications. However, in a typical MIMO system, using the same number of RF links as the antennas to ensure the normal communication of the MIMO system will greatly increase the complexity of the system and the cost of implementation, making the MIMO system much larger than the ordinary communication system, resulting in its limited application and promotion. The selection of antennas is therefore crucial, the core of which is to follow certain criteria for the selection of all antennas. Selecting a subset of multiple antennas can achieve high speed and high capacity communication, which can effectively reduce the number of RF links, thus simplifying the system and effectively improving the practicality of MIMO systems.

The optimal selection of antenna subset is the exhaustive algorithm $(\mathrm{EA})^{[6-7]}$, which calculates all possible combinations of antenna subsets, so as to select the subset that can make the system performance optimal. However, the EA algorithm also 
greatly increases the computational complexity, especially in the case of a large scale antennas the EA calculation time is too long, the computational volume is exponentially increasing, which means that it is difficult to obtain results in a limited time and finally reducing its practicality. In order to further improve the performance of the AS system, a joint transceiver antenna selection (JTRAS) algorithm is proposed ${ }^{[8]}$, which yields good results in terms of channel capacity. But the computational complexity of the algorithm is still very high and the performance is poor in terms of practicality. As a result, many scholars have proposed JTRAS algorithms ${ }^{[9-10]}$ to reduce the computational complexity of the algorithm from various aspects, which can still maintain a relatively high channel capacity while reducing the computational complexity of the algorithm. Alternatively, the AS is based on the principle of maximizing the minimum column parametrization of the channel matrix according to the maximum Frobenius parametrization ${ }^{[11]}$, which has no matrix operations and low computational complexity. A fast and global AS algorithm is proposed [12], in which the number of receiver antennas is the same as the number of transmit antennas. A real-time antenna-by-antenna iterative swapping enhancement is proposed based on the maximum capacity submatrix approach (RAISE) transmit antenna selection algorithm [13], but it also needs to be based on the premise that the number of antennas is equal to the number of users, meaning that they can only handle square submatrices, which is less realistic and practical. On this basis, the theory of rectangular maximum-volume (RMV) submatrices ${ }^{[14]}$ is proposed to eliminate the limitations of the traditional square maximum volume (SMV) antenna selection method, while maintaining the same good performance as the SMV method.A low-complexity AS scheme ${ }^{[15]}$, which maximizes the constructive interference between users and identifies a subset of antennas with the highest constructive interference, thus maximizing the power received by the user. Interestingly, Inspired by the branch-and-bound (BAB) search method for finding a subset of the beam, the BAB algorithm ${ }^{[16]}$ is also used to find a subset of antennas to maximize channel capacity(MCC), which shows three orders of magnitude higher than poor method when the number of antennas is large.

It can be observed that a suitable algorithm can help to solve the AS problem well, replacing the EA in reality. Intelligent algorithms are inspired by the laws of nature and designed to mimic their principles in solving problems. In recent years, intelligent algorithms have achieved numerous convincing results in solving practical problems, such as genetic algorithms (GA)for complex optimisation problems and industrial engineering applications, and particle swarm algorithms (PSO)which are now widely used in function optimisation, neural network training, and medicine. Thus, the combination of AS and intelligent algorithms is a new way of thinking and a different direction for exploring multiple AS techniques.

In this paper, a comprehensive comparison of three swarm intelligence algorithms, namely particle swarm algorithm (PSO), cat swarm algorithm (CSO) and genetic algorithm (GA) is presented. All three are heuristic algorithms, among which the GA is a simulation of the superiority and inferiority of biological populations in nature ${ }^{[12-13]}$, with the advantages of low computational complexity, good robusts, parallel search of the solution space, fast convergence, etc.; the $\mathrm{CSO}^{[14-15]}$ is derived from the observation of feline behaviour, involving few parameters, simple principles, fast convergence, and has better global detection and local search The PSO is a kind of evolutionary algorithm, which originates from the observation of the predatory behaviour of birds. The basic idea is to find the optimal solution through collaboration and information sharing between individuals in a group. The algorithm is designed with few parameters and is easy to implement with high accuracy. All three algorithms can be well integrated with the AS problem to solve realistic problems efficiently. Not only to this, we 
refer to the fractional coding (FC) method, which can be used in most intelligent algorithms because it can still encode and decode normally in the actual process without affecting the operation of the algorithm, and is more general than binary encoding and real encoding. Moreover, we specify how the three intelligent algorithms are nested with the antenna problem and analyze the characteristics and advantages of the three algorithms in this problem, with the aim of choosing the right algorithm according to the actual situation and needs.

\section{System model}

To greatly increase the channel capacity ${ }^{[1-2]}$, MIMO systems use multiple antennas at both the transmitter and receiver ends, and information is transmitted and received through multiple antennas between the transmitter and receiver ends. Without increasing spectrum resources and transmitting power, multi-antenna systems can increase the capacity of the system, improve the quality of the wireless transmission link, and increase the bandwidth of the system exponentially and reliability. Assuming a MIMO system with $\mathrm{N}_{\mathrm{t}}$ transmitting antennas and $\mathrm{N}_{\mathrm{r}}$ receiving antennas, at the transmitting end, the transmitted information stream is coded in space-time to form $\mathrm{N}_{\mathrm{t}}$ identical data sub-streams, which are transmitted simultaneously from $\mathrm{N}_{\mathrm{t}}$ antennas and received by $\mathrm{N}_{\mathrm{r}}$ antennas after passing through the spatial channel, the multi-antenna receiver is able to separate and decode these data sub-streams using space-time coding, thus achieving spatial diversity gain. Because the receiver obtains multiple independent signal copies of the same information from multiple channel bearers, and the signals are not in deep fading at the same time, so at least one copy of the signal with sufficient strength is guaranteed to be accepted at any given moment, then the receiver can achieve bigger signal-to-noise ratio (SNR) than single antenna.

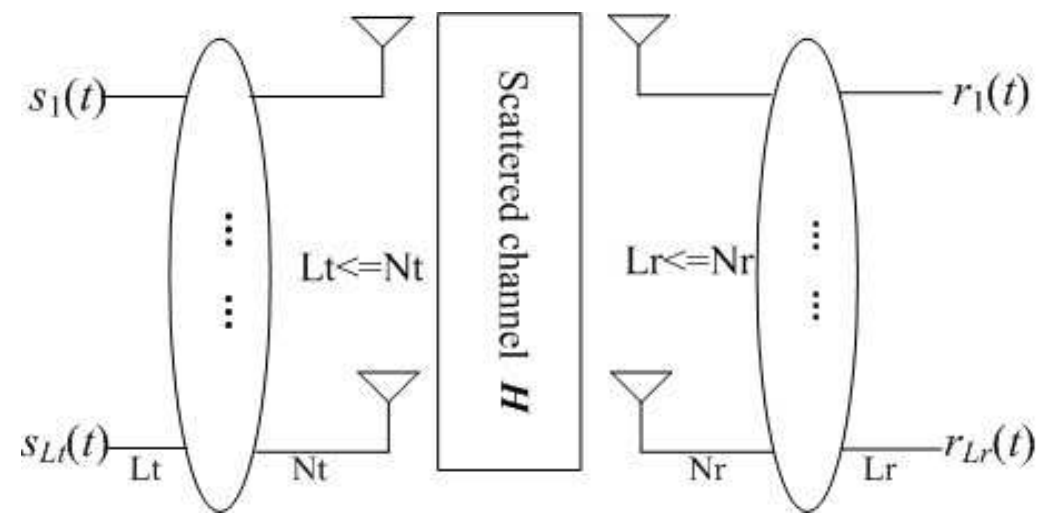

Figure 1. System model

However, as many RF links are used to ensure proper communication in a MIMO system, it will increase the complexity and cost of the system. Hence, AS is a key technology that can significantly reduce processing complexity and hardware costs while maintaining the benefits of MIMO technology. As shown in figure 1, let $\mathrm{L}_{t}$ and $\mathrm{L}_{\mathrm{r}}$ denote the subset of antennas selected from $N_{t}$ and $N_{r}$ by the AS algorithm, satisfying $N_{t} \geq L_{t}$ and $N_{r} \geq L_{r}$. In addition, $\boldsymbol{H}$ is a random channel matrix, denoted as the unselected $\mathrm{N}_{t} \times \mathrm{N}_{\mathrm{r}}$ channel matrix, assuming that the symbols transmitted at each antenna cell are uncorrelated and have normalized power. In the case of frequency-flat fading, the signal at the receiver can be expressed as

$$
\begin{aligned}
\boldsymbol{R}=\left[\begin{array}{c}
r_{1} \\
r_{2} \\
\vdots \\
r_{\mathrm{L}_{\mathrm{r}}}
\end{array}\right] & =\sqrt{\frac{\rho}{\mathrm{L}_{\mathrm{t}}}}\left[\begin{array}{cccc}
h_{11} & h_{12} & \cdots & h_{\mathrm{L}_{\mathrm{t}}} \\
h_{21} & h_{22} & \cdots & h_{2 \mathrm{~L}_{\mathrm{t}}} \\
\vdots & \vdots & \ddots & \vdots \\
h_{\mathrm{L}_{\mathrm{r}} 1} & h_{\mathrm{L}_{\mathrm{r}} 2} & \cdots & h_{\mathrm{L}_{\mathrm{r}} \mathrm{L}_{\mathrm{t}}}
\end{array}\right]\left[\begin{array}{c}
r_{1} \\
r_{2} \\
\vdots \\
r_{\mathrm{L}_{\mathrm{t}}}
\end{array}\right]+\left[\begin{array}{c}
w_{1} \\
w_{2} \\
\vdots \\
w_{\mathrm{L}_{\mathrm{r}}}
\end{array}\right] \\
& =\sqrt{\frac{\rho}{\mathrm{L}_{\mathrm{t}}}} \boldsymbol{H} \boldsymbol{S}+\boldsymbol{w}
\end{aligned}
$$

Where $\boldsymbol{R}$ is receiving signal vector, $\boldsymbol{S}$ is the transmitting signal vector, $\rho$ is the average signal-to-noise ratio of each 
transmitting antenna of the transmitter, and W is the additive Gaussian white noise (AWGN) with a variance of 1/2 in both the real and imaginary parts.

The system capacity is one of the most important signs characterizing a communication system and represents the maximum transmission rate of the communication system. The channel capacity of a MIMO system is expressed as

$$
\mathrm{C}=\operatorname{Ibdet}\left(\boldsymbol{I}_{L r}+\frac{\rho}{L_{t}} \boldsymbol{H}_{L_{r} \times L_{t}} \boldsymbol{H}_{L_{r} \times L_{4}}^{\mathrm{H}}\right)
$$

where $\boldsymbol{I}$ is the unit matrix of $L_{t} \times L_{r},(\cdot)^{\mathrm{H}}$ is the conjugate transpose, $\operatorname{det}(\cdot)$ denotes taking the determinant, and cell $h_{\mathrm{ij}}$ is the channel fading coefficient from transmit antenna $j$ to receive antenna $i$ in the channel matrix $\boldsymbol{H} . h_{\mathrm{ij}}$ is independently distributed and satisfies a Gaussian distribution with mean zero variance of $1 / 2$. The channel capacity C is numerically equal to the channel efficiency (bits/Hz). There is sufficient fading in the channel and the envelope conforms to the Rayleigh distribution. The aim of antenna selection is therefore to select a new channel matrix from $\boldsymbol{H}$ so as to maximize the channel capacity.

\section{Swarm intelligence algorithm design}

\subsection{Genetic coding}

Most of the coding methods in AS are binary coding, e.g., the use of 0,1 mask for antenna coding, but in the process of swarm intelligence algorithm, the number of antennas required is different. Hence, it is difficult to ensure the certain number of antennas to be selected. To tackle with the problem, this paper investigates fractional coding (FC), which can avoid the number of antennas that does not correspond to the problem, to ensure the general operation. Specifically, a random decimal encoding of 0 to 1 is used for $N_{t}+N_{r}$ antennas, and the number indices are selected from largest to smallest, in accordance with the number of antennas required. Take the following GA as an example, we set $N_{t}=4, N_{l}=3, L_{l}=2, L_{l}=2$ : figure 2 shows a chromosome encoding, the number of genes depends on the total number of antennas, the first $N_{t}$ bits indicate the transmitter antennas, the last $N_{r}$ bits indicate the receiver antennas. Given a random decimal number and then arranged from highest to lowest, $L t$ and $L r$ are selected according to the requirement of 2 out of 4 chosen from the transmitter, then antennas $\{2,4\}$ are selected, and 2 out of 3 from the receiver, then $\{1,2\}$ are selected.

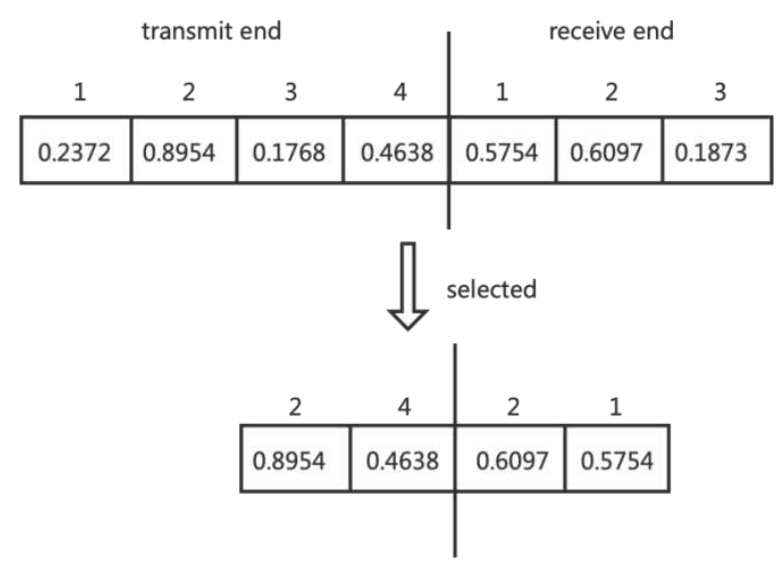

Figure 2. Chromosome encoding

The following figure shows an example of a crossover operation of a GA to better understand FC. 


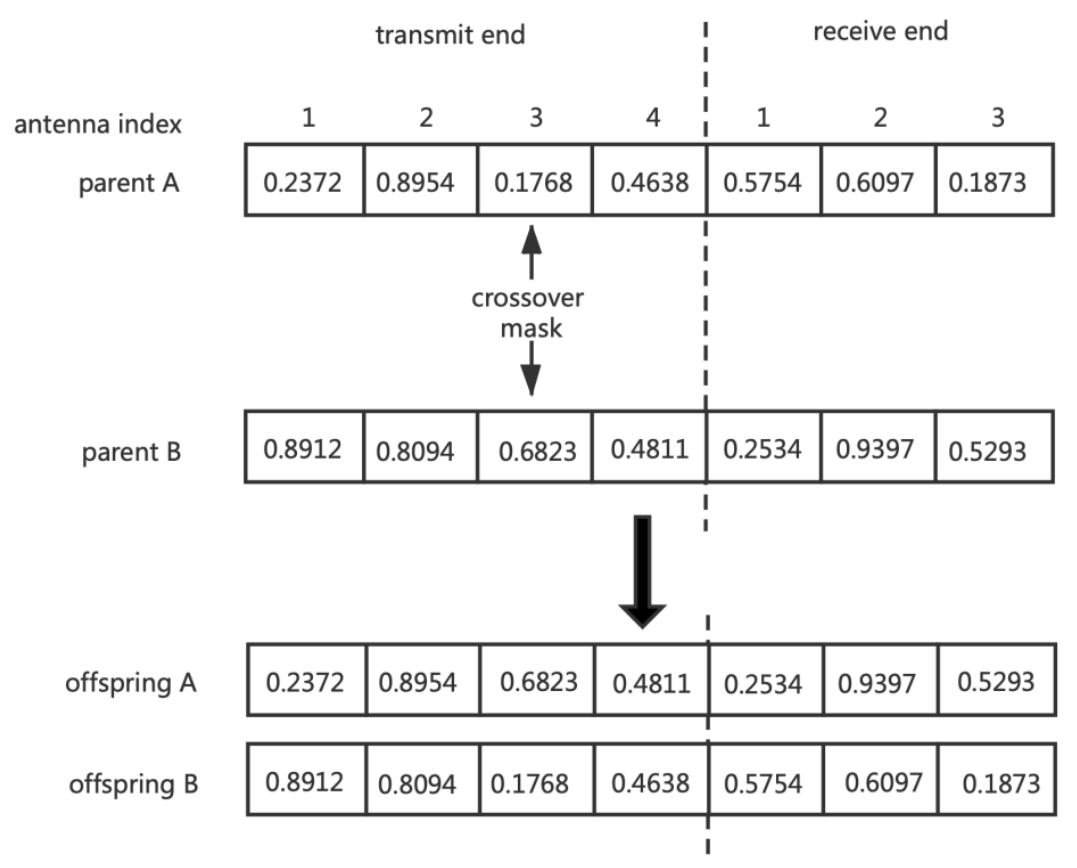

Figure 3. Crossover operation of chromosome

In this paper, three intelligent algorithms are used to solve the AS problem.

\subsection{Genetic algorithms}

Firstly, the genetic algorithm(GA), as a class of randomised search method that simulates the evolutionary laws of the biological world, is a bionic algorithm with a strong global search for superiority, in which more individuals with higher fitness are passed on to the next generation according to the rules of superiority and inferiority, so that some individuals with lower fitness in the population are gradually eliminated, while some individuals with higher fitness become more and more numerous. The process of AS based on genetic algorithm is shown in figure 4.

Step 0: Setting the parameters. Firstly, the relevant parameters required for the algorithm should be set, such as the number of chromosomes, the number of iterations of the algorithm, the number of transmitting and receiving antennas of the MIMO system, the number of antennas selected for the target, the crossover probability, the variation probability, etc.

Step 1: Initialization: Initialize the parent population, the size of the population is popsize. Each chromosome represents a combination of antennas, and the number of genes contained in the chromosome is the sum of the number of antennas at the sending and receiving ends, i.e. $N_{r}+N_{t}$. Each gene is a random decimal number between 0 and 1 , and the decimal numbers are sorted from largest to smallest to take the corresponding position. Each chromosome corresponds to a set of selected antennas.

Step 2: Adaptation evaluation and chromosome selection replication. Each chromosome corresponds to a set of antennas, and each set of selected antennas corresponds to a channel matrix $\boldsymbol{H}_{\mathrm{s}}$.

Step 3: Chromosome crossover operation. In this paper, a single point crossover is used to generate a random crossover point in the chromosome string, and when the crossover is implemented, the two genes after that point are swapped according to a certain probability to generate a new chromosome string. 
Step 4: Chromosomal variation operation. The probability of variation is such that a random number between 0 and 1 is generated at the point of variation in place of the original gene.

Step 5: Update the population. The chromosome adaptations in the gene pool are calculated after crossover and mutation, and the chromosome with the highest adaptation is found in the population and compared with the maximum adaptation of the chromosome in the original population, if it is larger, the chromosome with the highest adaptation in the population is replaced with the newly generated chromosome in the pairing pool, and vice versa. The new population is obtained by repeated iteration through all the chromosomes generated in the pool.

Step 6: Repeat/end: Repeat steps 2-5 above until the number of iterations is reached. The chromosome with the greatest fitness in the final population is the optimal antenna combination sought. Finally, the number of antennas at both transmitter and receiver is decoded according to the required selection, resulting in the $L_{t}$ transmit antenna and $L_{r}$ receive antenna with excellent performance.

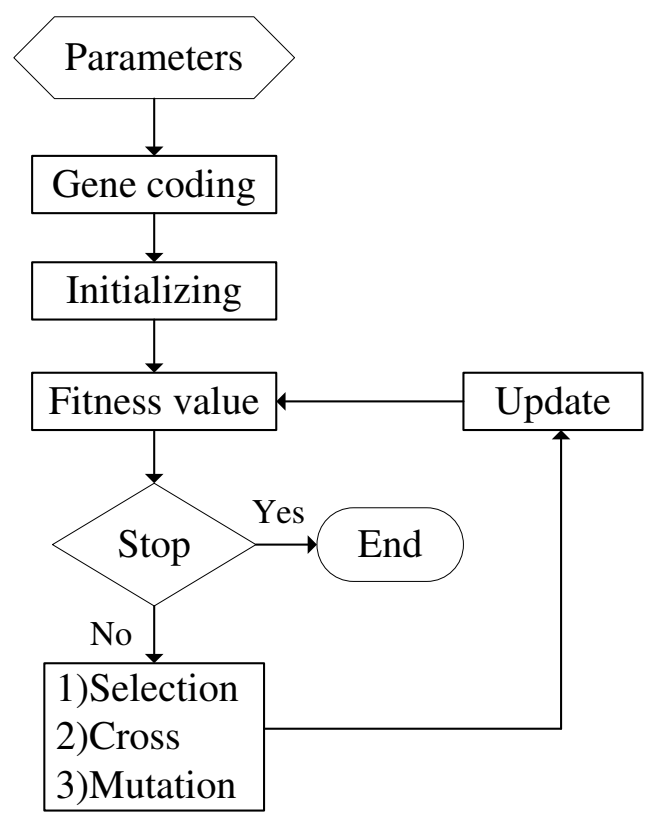

Figure 4. AS based on GA

\subsection{Cat swarm optimization}

The cat swarm optimization (CSO) is a group intelligence bionic algorithm proposed by observing the behavior of cats, each of which represents a combination of antennas. The algorithm divides the behavior of the cats into search mode, whereby the cats are resting and looking, and tracking mode, whereby the cats are tracking a dynamic target. Different interaction rates are set to randomly assign cats to the search and tracking modes. In the search mode, the memory pool records the neighborhood searched by the cat, the size of the pool represents the number of locations the cat can search, and the pool stores the new locations that the cat can search in its own neighborhood. The location code corresponding to the largest fitness value is selected to replace the current location value of the corresponding cat. In the tracking mode, in each iteration, the cat will track an 'extreme value' to update itself, which is the optimal solution found by the whole population, so that the cat moves in the direction of the global optimal solution. The algorithm involves few parameters, reaches convergence values quickly and has excellent global detection as well as local search capabilities. The AS process based on the cat swarm optimization is shown in figure 5 : 
Step 0: Set the parameters required for the algorithm: in addition to the population size of the cat population, the number of iterations of the algorithm, the number of transmitting and receiving antennas of the MIMO system, the number of antennas selected for the target, the parameters required in the search mode such as the memory pool, the change domain, etc., the interaction rate, etc.

Step 1: Initialise and calculate the fitness value: each cat represents an antenna combination, then each cat's position is coded as $\left(\mathrm{N}_{\mathrm{t}}+\mathrm{N}_{\mathrm{r}}\right)$ fractional codes, and these cats are randomly assigned to the tracking mode and the searching mode according to the interaction rate.

Step 2:Update the speed and position: according to the two modes each cat is in, update the position and speed.

Step 3:Calculate the new fitness value. The largest fitness value in the population is the new optimal solution, if it is larger than the previous optimal solution then the new fitness value is replaced with the new optimal solution.

Step 4: Judgment condition: if the optimal solution is satisfied or the set number of iterations has been reached, the global optimal solution is output and finished; otherwise, repeat step 2-3 to continue the search for the optimal solution.

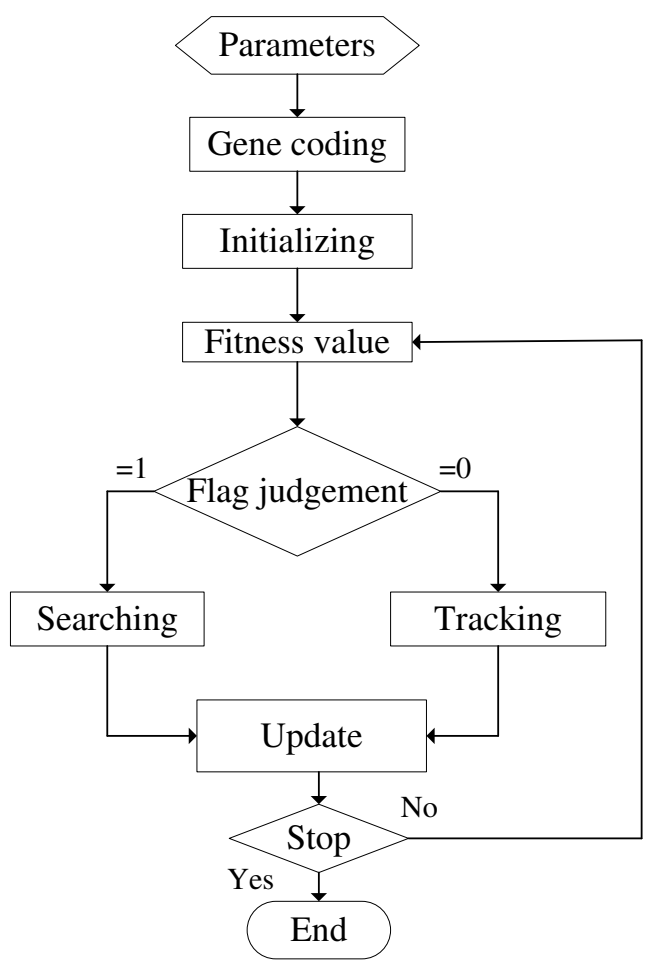

Figure 5. AS based on CSO

\subsection{Particle swarm algorithms}

The particle swarm algorithm (PSO) simulates the predatory behavior of a flock of birds. PSO is essentially a stochastic search algorithm and an optimization algorithm based on population intelligence theory, so PSO can converge to the global optimum with a higher probability. Compared to GA, PSO retains the population-based global search strategy and uses a simple velocity-displacement model without the need for complex genetic operations. However, in the case of convergence, as all particles converge to the optimal solution, the particles tend to homogenize making the convergence rate significantly slower in the later stages. The AS process based on the particle swarm algorithm is shown in figure 6: 
Step 0: Set parameters. Set the relevant parameters required by the algorithm, in addition to those mentioned above at the beginning there are acceleration constants $c_{1}, c_{1}$, inertia weights $\omega$, etc.

Step 1: Initialise and calculate the fitness value. initialise the velocity and position of the particles, where each particle position and velocity is represented by a decimal code and each particle represents a combination of antennas, then calculate the fitness value of all the particles in the population and compare the fitness value of each particle with the individual extreme value, and replace the original individual extreme value if it is larger; then compare the fitness value of each particle with the global extreme value, and replace the original global extreme value if it is larger. The global polarity is then replaced by the fitness value of each particle if it is larger.

Step 2: Update the velocity and position of the particle according to (3) and (4), where $P_{i}$ is the individual extremum, $P_{g}$ is the global extremum, $\mathrm{c}_{1}, \mathrm{c}_{2}$ is the learning factor, and $\mathrm{r}_{1}, \mathrm{r}_{2}$ is a uniform random number in the range $[0,1]$.

$$
\begin{gathered}
v=\omega \cdot v+\mathrm{c}_{1} \mathrm{r}_{1}\left(p_{i}-x\right)+\mathrm{c}_{2} \mathrm{r}_{2}\left(p_{\mathrm{g}}-x\right) \\
x=x+v
\end{gathered}
$$

Step 3: Judgment. If the optimal solution is satisfied or the set number of iterations has been reached, the global optimal solution is output and finished; otherwise, repeat step 2 to continue the search.

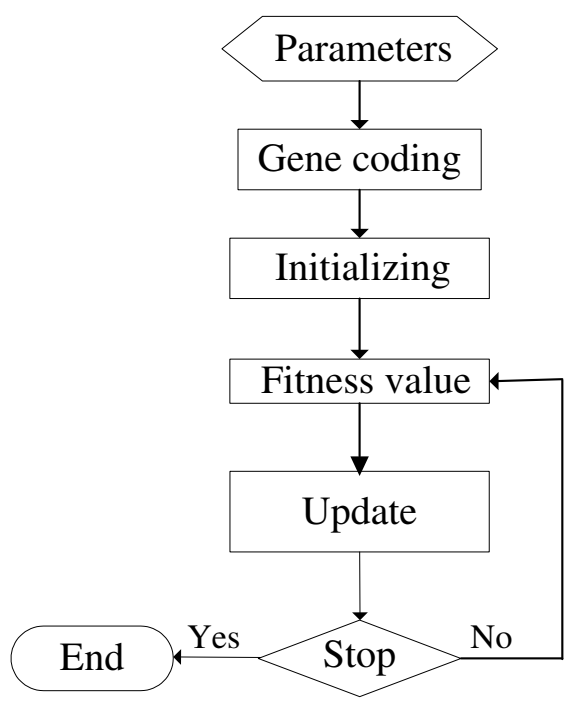

Figure 6. AS based on PSO

\section{Simulation and analysis}

This section will analyze the simulation results of the three algorithms, with parameters all set to: signal-to-noise ratio SNR = 4; number of populations, popsize $=20$; number of iterations $\mathrm{G}=200 ; \mathrm{N}_{\mathrm{t}}=10 ; \mathrm{N}_{\mathrm{r}}=10 ; \mathrm{L}_{\mathrm{r}}=5 ; \mathrm{L}_{\mathrm{r}}=5$. From figure 7, we can see that all three algorithms can be well applied in the AS problem, and as the number of iterations increases, the fitness values all show the upward trend. 


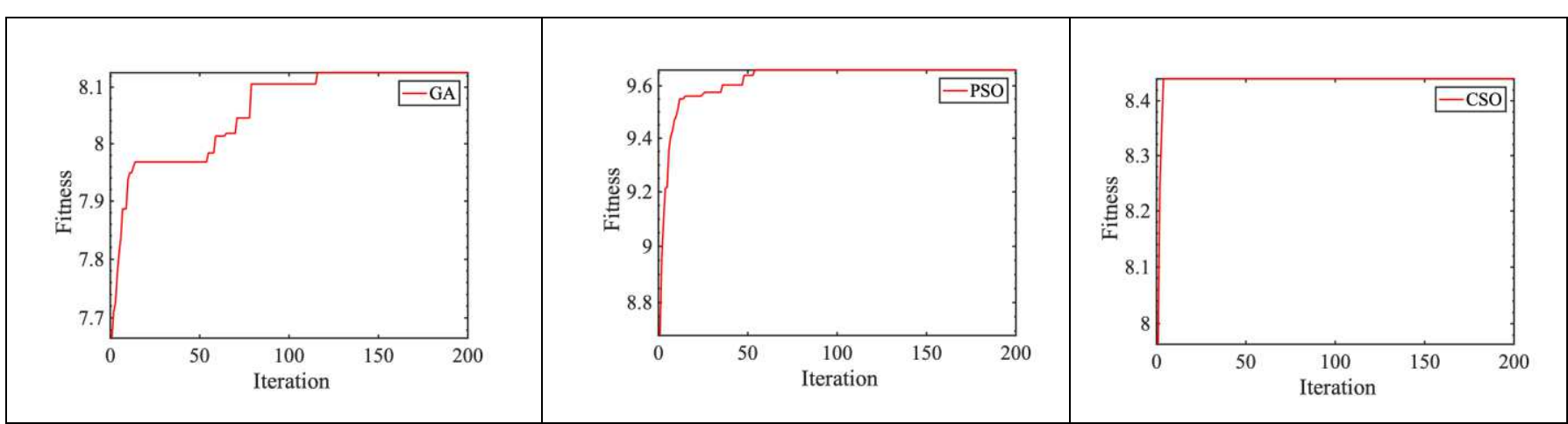

Figure 7. Fitness v.s. iteration

By comparing the maximize channel capacity (MCC) versus SNR in figure 8, it can be seen that the best values obtained by all three algorithms and the optimal EA are very close, and the MCC will increase with the SNR.

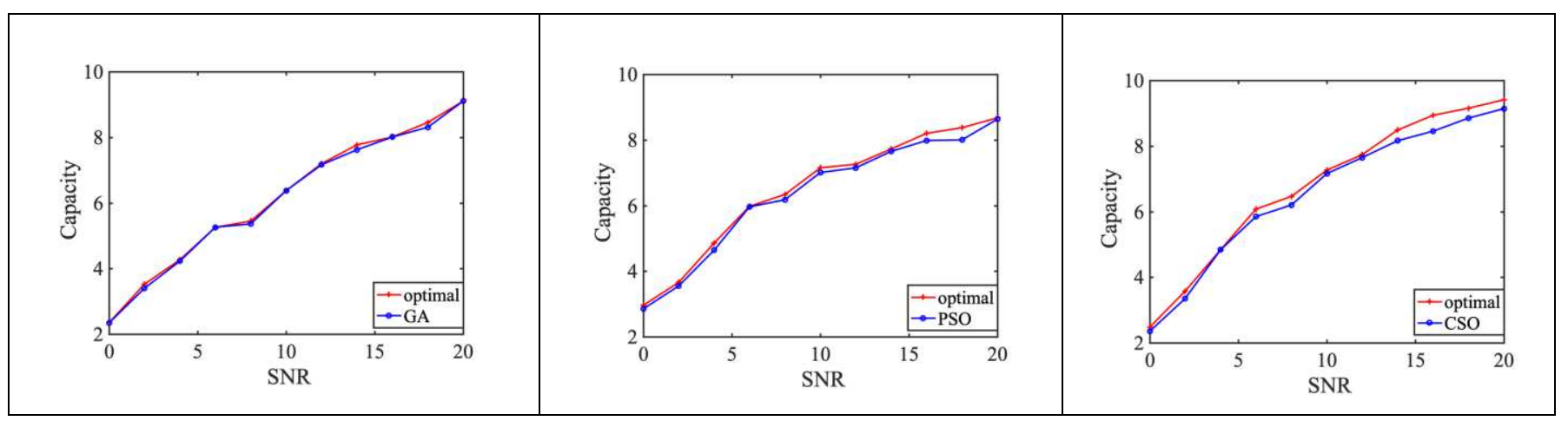

Figure 8. Capacity v.s. SNR

We would like to investigate above four algorithms in terms of accuracy, speed of finding the optimum, stability and complexity. The results obtained by the exhaustive enumeration method are regarded as the optimal solution. Set the initial value of the number of iterations $\mathrm{G}=100$ and the number of populations, pop size $=20$, then run the simulation 20 iterations to obtain the results.

The accuracy is defined as the ratio of the maximum value of the channel capacity for the intelligent algorithm to the EA solution, and the table shows that the accuracy of the three algorithms to solve the AS problem of the MIMO system from largest to smallest is PSO > CSO > GA, in which the accuracy of PSO is above $90 \%$ for all three antenna numbers, and the accuracy is similar in the cases of 10 and 15 transmit-and-receive antennas. The accuracy of both CSO and GA decreases significantly with the increase of the number of antennas, and it is also obvious that PSO has higher accuracy compared with the other two algorithms. Therefore, PSO is much close to the optimal solution in solving the AS problem.

Table 1 Accuracy of intelligent algorithms

\begin{tabular}{lccc}
\hline & $\mathrm{Nt}=5 \mathrm{Nr}=5 \mathrm{Lt}=2 \mathrm{Lr}=2$ & $\mathrm{Nt}=10 \mathrm{Nr}=10 \mathrm{Lt}=4 \mathrm{Lr}=4$ & $\mathrm{Nt}=15 \mathrm{Nr}=15 \mathrm{Lt}=5 \mathrm{Lr}=5$ \\
\hline CSO & $95.76 \%$ & $90.52 \%$ & $87.02 \%$ \\
PSO & $98.05 \%$ & $91.86 \%$ & $92.29 \%$ \\
\hline
\end{tabular}




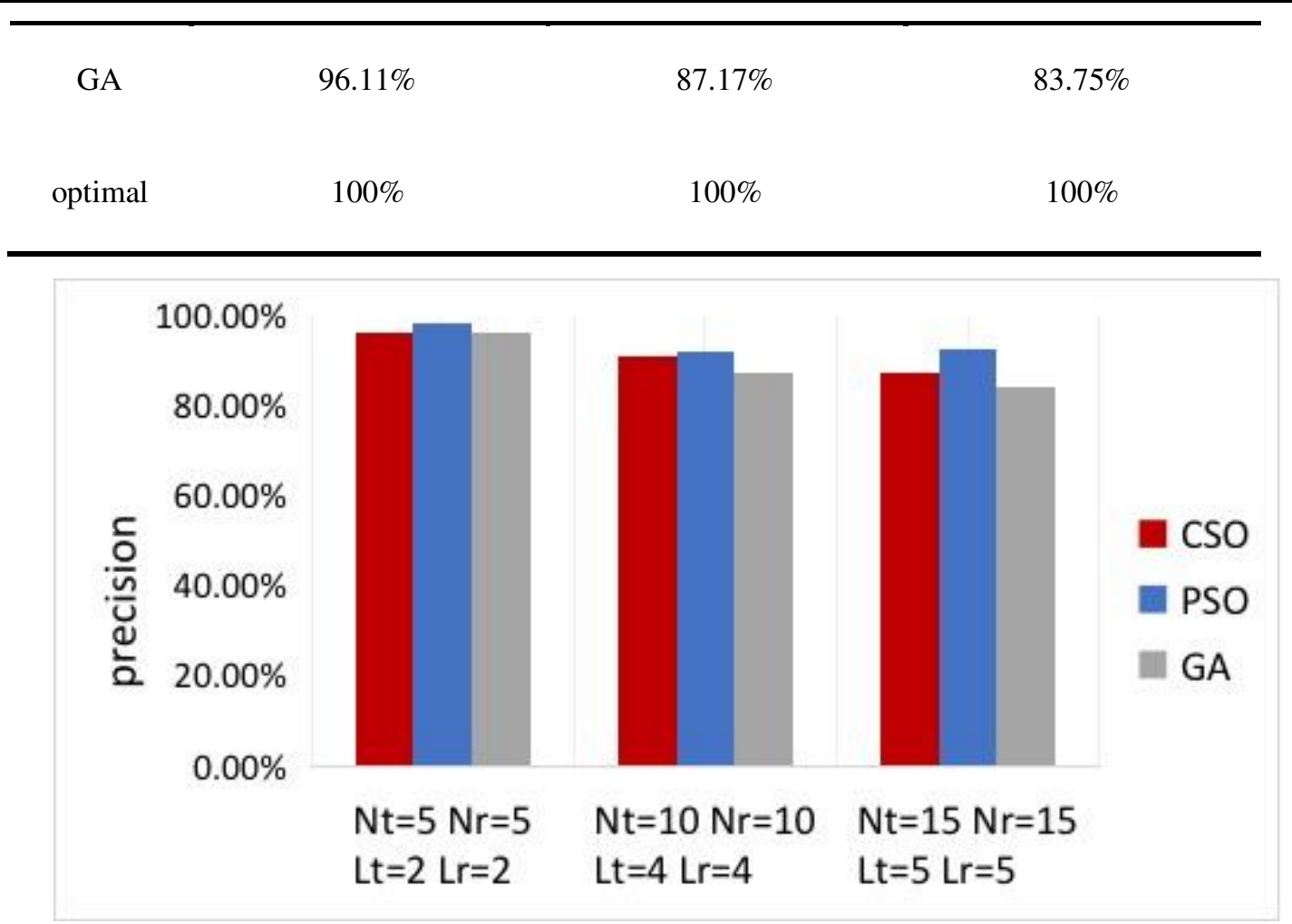

Figure 6. Precision of swarm intelligence algorithms

The stability is defined as the standard deviation of the optimal solution for 20 consecutive runs, and a small standard deviation means that the algorithm is the most stable. Table 2 shows that the standard deviation obtained by PSO is lower than that obtained by the other two algorithms, indicating that PSO is able to obtain the optimal value with a greater probability and with a smaller error. The stability of the other two algorithms is similar.

Table 2 Stability of intelligent algorithms

\begin{tabular}{lccc}
\hline & $\mathrm{Nt}=5 \mathrm{Nr}=5 \mathrm{Lt}=2 \mathrm{Lr}=2$ & $\mathrm{Nt}=10 \mathrm{Nr}=10 \mathrm{Lt}=4 \mathrm{Lr}=4$ & $\mathrm{Nt}=15 \mathrm{Nr}=15 \mathrm{Lt}=5 \mathrm{Lr}=5$ \\
$\mathrm{CSO}$ & 0.043 & 0.035 & 0.039 \\
PSO & 0.021 & 0.034 & 0.016 \\
GA & 0.039 & 0.046 & 0.045 \\
\hline
\end{tabular}

The search speed is defined as the number of iterations to reach convergence for the first time, and the comparison shows that the number of iterations required to reach convergence increases with the number of antennas for all three algorithms. The speed of the search is from fast to slow is CSO $>$ GA $>$ PSO, especially the advantage of CSO in the speed of the search is very obvious, compared to the slowest speed of PSO.

Table 3 Search speed of Intelligent algorithms 


\begin{tabular}{lccc}
\hline & $\mathrm{Nt}=5 \mathrm{Nr}=5 \mathrm{Lt}=2 \mathrm{Lr}=2$ & $\mathrm{Nt}=10 \mathrm{Nr}=10 \mathrm{Lt}=4 \mathrm{Lr}=4$ & $\mathrm{Nt}=15 \mathrm{Nr}=15 \mathrm{Lt}=5 \mathrm{Lr}=5$ \\
\hline $\mathrm{CSO}$ & 2.13 & 4.06 & 4.31 \\
PSO & 22.31 & 35.25 & 47.94 \\
GA & 18 & 31.6 & 38 \\
\hline
\end{tabular}

In terms of complexity, the EA requires calculations to $C_{N_{t}}^{L_{t}} \times C_{N_{r}}^{L_{r}}$ complete one AS, while the complexity of the remaining three algorithms is related to the population size and the number of iterations at convergence, i.e. complexity=popsizexiter. The table below shows that the complexity of the three algorithms is much lower than that of the EA, which means that all three algorithms greatly reduce the complexity of operation in JTRAS, In addition, the CSO has the lowest complexity among the three algorithms.

Table 4 Complexity of intelligent algorithms

\begin{tabular}{lccc}
\hline & $\mathrm{Nt}=5 \mathrm{Nr}=5 \mathrm{Lt}=2 \mathrm{Lr}=2$ & $\mathrm{Nt}=10 \mathrm{Nr}=10 \mathrm{Lt}=4 \mathrm{Lr}=4$ & $\mathrm{Nt}=15 \mathrm{Nr}=15 \mathrm{Lt}=5 \mathrm{Lr}=5$ \\
$\mathrm{CSO}$ & 43 & 81 & 86 \\
PSO & 446 & 705 & 769 \\
GA & 360 & 632 & 760 \\
EA & & & $>9.0 \times 10^{6}$ \\
\hline
\end{tabular}

Table 5 Performances of intelligent algorithms

\begin{tabular}{cccc}
\hline & CSO & PSO & GA \\
\hline Accuracy & Good & Excellent & Fair \\
Speed & Excellent & Fair & Good \\
Complexity & Low & High & Average \\
Stability & Fair & Excellent & Good \\
\hline
\end{tabular}




\section{Conclusions}

This paper investigates three intelligent algorithms, GA, CSO and PSO in joint transmit and receive antenna selection (JTRAS) for MIMO system. We employ the fractional coding (FC) method in the chromosome gene. The simulation results show that the PSO has the highest accuracy and stability, but the search speed is slower and the complexity is higher; the CSO has the fastest search speed and the lowest complexity, and the accuracy is worse compared with the PSO. However, the complexity of all three algorithms is much lower than that of the exhaustive enumeration method, so the appropriate algorithm can be used according to the actual needs.

In the future, large-scale MIMO systems will serve as an option for beyond 5G. Since the number of antennas in large-scale MIMO systems may be dozens or hundreds, intelligent algorithms should play more inportant role for JTRAS in MIMO systems.

\section{Acknowledgements}

The authors would like to thank the reviewers for their valuable comments for this paper. This work is supported in part by the Natural Science Foundation of Fujian Province (2020J01711), and Research Start-up Fund of Jimei University (ZQ2019021).

\section{Declarations}

- Funding: This work is supported in part by the Natural Science Foundation of Fujian Province (2020J01711), and Research Start-up Fund of Jimei University (ZQ2019021) both with the ownership of Lei Guowei.

- Conflicts of interest/Competing interests: No conflicts of interests.

- Availability of data and material: Applicable.

- Code availability: Not applicable.

- Authors' contributions: Sun Sunqing proposed the theoretical method. Liao Wenliang gave the program flowcharts. Zhang Yiwen wrote the program codes and ran the simulations. Zhang Yiwen and Lei Guowei wrote the main manuscript. Yang Guangsong reviewed the paper and gave some corrections.

\section{References}

[1] Marzetta T.L,Hochwald B.M. Capacity of a mobile multiple-antenna communication link in Rayleigh flat fading[J]. IEEE Transactions on information theory, 1999, vol. 45, no.1, pp.139-157.

[2] Lei G.W., Liu Y.A., Xiao X.F. Evaluation of bit error probability for CPM MIMO systems in Rayleigh channel [J]. Wireless personal communications, 2015, vol. 85, no.3, pp. 585-595.

[3] Mietznerj S., Lampel L, et al. Multipie antenna techniques for wireless communications: a comprehensive literature survey [J].IEEE Communications surveys \& tutorials, 2009, vol. 11, no.2, pp.87-105. 
[4] Molisch A.F., Win M.Z. MIMO systems with antenna selection [J]. IEEE Microwave magazine, 2004, vol.5, no.1, pp.46-56.

[5] Paulraja J, Gored A, Nabar R. U, Bolcskei H. An overview of MIMO communications-a key to gigabit wireless [J]. Proceedings of the IEEE, 2004, vol.92, no. 2, pp.198-218.

[6] Sanayei S., Nosratinia A. Antenna selection in MIMO systems [J]. IEEE Communications magazine, 2004, pp. 42 , no. 10 , pp.68-73.

[7] Andreas F.M., Moe Z.W., Jack H.W. Capacity of MIMO systems with antenna selection [C]. IEEE International conference on communications. Helsinki, Finland, 2001, pp. 570-574.

[8] Gorokhov A., Collados M. Gore D., et al. Transmit/receive MIMO antenna subset selection[C]. IEEE International conference on acoustics, speech, and signal processing. Montreal, Canada, 2004, pp.13-16.

[9] Blum R.S., Xu Z., Sfar S. A near optimal joint transmit and receive antenna selection algorithm for MIMO systems [C]. Proceedings of radio and wireless Symposium, San Diego, USA, 2009, pp.554-557.

[10] Naeem M., LEE D.C. Near optimal joint selection of transmit and receive antennas for MIMO systems[C]. 2009 IEEE International Symposium on Communications and Information Technology. South Korea, 2009:572-577.

[11] Wang Y., Song J., Ge L.D. A combined antenna selection algorithm in MIMO system[C]. 2007 International conference on microwave and millimeter wave technology, Guilin, 2007, 1-4.

[12] Wang B.H., Hui H.T., Leong M.S. Global and fast receiver antenna selection for MIMO systems. IEEE Transactions on communications, 2010, vol. 58, no. 9, pp. 2505-2510.

[13] Fang $\quad$ B., $\quad$ Qian $\quad$ Z.P., Shao $\quad$ W., $\quad$ Zhong $\quad$ W. $\quad$ RAISE: A new fast transmit antenna selection algorithm for massive MIMO systems. Wireless personal communications, 2015, vol. 80 , no. 3 , pp. $1147-1157$.

[14] Tang H., Nie Z.P. RMV antenna selection algorithm for massive MIMO. IEEE Signal processing letters, 2018, vol. 25, no. 2, pp. 39-42.

[15] Amadori P.V., Masouros C. Interference-driven antenna selection for massive multiuser MIMO[J]. IEEE Transactions on vehicular technology, 2016, vol. 65, No. 8, pp. 5944 - 5958.

[16] Gao Y., Vinck H., Kaise T. Massive MIMO antenna selection: switching architectures, capacity bounds, and optimal antenna selection algorithms [J]. IEEE Transactions on signal processing, 2018, vol. 66, no. 5, pp.1346-1360.

[17] Makki B. Ide A, Svensson T, et al. A genetic algorithm based antenna selection approach for large but finite MIMO networks [J]. IEEE Transactions on vehicular technology, 2017, vol.66, no.7, pp.1-5.

[18] Lu H.Y., Fang W.H. Joint transmit/receive antenna selection in MIMO systems based on the priority-based 
genetic algorithm [C]. Proceedings of IEEE antennas and wireless propagation letters, 2007, 6, pp. 588-591.

[19] Sharafi Y., Khanesar M.A, Teshnehlab M. Binary cat swarm optimization algorithm[C]. 3rd International conference on computer, control \& communication, 2013, pp. 1-6.

[20] Zhuang M.J, Zhuang H. Application of binary cat swarm optimization in large-scale MIMO antenna selection [J]. Telecommunication engineering, 2017, vol. 57, no.6, pp. 698-702. 